\title{
Atomic Resolution Imaging of Rotated Bilayer Graphene Sheets Using a Low kV Aberration-corrected Transmission Electron Microscope
}

\author{
Gyeong Hee Ryu, Hyo Ju Park, Na Yeon Kim, Zonghoon Lee* \\ School of Mechanical and Advanced Materials Engineering, Ulsan National Institute of Science and Technology (UNIST), \\ Ulsan 689-798, Korea
}

*Correspondence to:

Lee $\mathrm{Z}$,

Tel: +82-52-217-2327

Fax: +82-52-217-2309

E-mail: zhlee@unist.ac.kr

Received November 30, 2012

Revised December 3, 2012

Accepted December 4, 2012
Modern aberration-corrected transmission electron microscope (TEM) with appropriate electron beam energy is able to achieve atomic resolution imaging of single and bilayer graphene sheets. Especially, atomic configuration of bilayer graphene with a rotation angle can be identified from the direct imaging and phase reconstructed imaging since atomic resolution Moiré pattern can be obtained successfully at atomic scale using an aberrationcorrected TEM. This study boosts a reliable stacking order analysis, which is required for synthesized or artificially prepared multilayer graphene, and lets graphene researchers utilize the information of atomic configuration of stacked graphene layers readily.

Key Words: Bilayer graphene, Aberration-corrected TEM imaging, Atomic resolution, Low $\mathrm{kV}$ imaging, Simulation

\section{INTRODUCTION}

Graphene, a single layer of $\mathrm{sp}^{2}$-bonded carbon structure, presents new opportunities in fundamental research and practical applications because of its extraordinary and unique electrical, mechanical, and thermal properties (Novoselov et al., 2004).

Bilayer graphene, which consists of two graphene layers lying one on the other, has a great attraction along with multilayer structures because the properties of bilayer graphene and even more layers can be modified significantly to achieve unique characteristics not available in single layer graphene (Ohta et al., 2006; Abergel \& Fal'ko, 2007; Castro Neto et al., 2009). Changing stacking order of graphene layer varies two-dimensional atomic structure and it brings new characteristics. Many graphene researchers usually point out no bandgap opening in graphene limits the usage of graphene in electronics, but Zhang et al. (2009) at U.C. Berkeley revealed bilayer graphene gets a tunable bandgap at room temperature to make a transistor. In general as monolayer graphene, bilayer graphene also has a zero bandgap and thus behaves like a semimetal. But they proposed a bandgap can be introduced if the mirror-like symmetrical structure of the two layers is disturbed, the bilayer graphene then behaves like a semiconductor (Ohta et al., 2006). And researchers at U.C. Riverside reported Bernal-stacked trilayer graphene can alter electrical properties of graphene, which makes them from conductor to insulator by changing stacking and shifting of graphene layer structure each other (Bao et al., 2011).

Thus indentifying stacking structure of bilayer and multilayer graphene is quite essential to study the unique properties and applications of multilayer graphene. Especially, the atomic structure of bilayer, one of simplest cases, needs to be understood properly at atomic scale to extract the relation between its properties and production methods. Here we report the atomic structure identification of freestanding bilayer graphene in detail thanks to the most recently developed aberration-corrected transmission

This work was supported by Mid-career Researcher Program and Nano Material Technology Development Program through the National Research Foundation of Korea (NRF) funded by the Ministry of Education, Science and Technology (No.2011-0029412) and (2012M3A7B4049807).

@ This is an open-access article distributed under the terms of the Creative Commons Attribution Non-Commercial License (http://creativecommons.org/licenses/by-nc/3.0) which permits unrestricted noncommercial use, distribution, and reproduction in any medium, provided the original work is properly cited.

Copyright (c) 2012 by Korean Society of Microscopy 


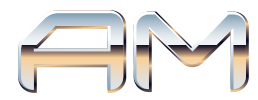

electron microscope (TEM) operating at a low $\mathrm{kV}$, which can achieve single atom detection of light elements like carbon in the lattice. Most ultra-high resolution TEM investigations of graphene have been focused on single layer graphene itself and defects such as grain boundaries, point defects and folding of graphene layers (Banhart et al., 2011; Kim et al., 2011a, 2011b). Furthermore, the aberration-corrected imaging at a low $\mathrm{kV}$ is able to achieve the imaging of the interface of soft-hard matter (Lee et al., 2009a). However, the resolving power and sensitivity to carbon atoms can be extended to investigation of the atomic stacking structure of bilayer and multilayer graphene sheets (Lee et al., 2009b).

\section{MATERIALS AND METHODS}

The free-standing graphene sheets, i.e. a two-dimensional single atomic sheet of carbon (Novoselov et al., 2005), are synthesized successfully from ethanol in a microwave plasma reactor (Dato et al., 2008) and from $\mathrm{CH}_{4}$ gas as a carbon source at $1,000^{\circ} \mathrm{C}$ in a thermal chemical vapor deposition ( $\mathrm{Li}$ et al., 2009; Park \& Ruoff, 2009). Both cases, bilayer graphene can be found. The synthesized graphene sheets are transferred to Quantifoil carbon grid (Quantifoil Micro Tools GmbH, Jenna, Germany) for TEM observation. The Titan Cube (FEI Company, Eindhoven, The Netherlands) is equipped with $\mathrm{C}_{\mathrm{s}}$ image aberration corrector for illumination and projection systems and with an electron monochromator, which have been optimized in range of operating voltage $60 \sim 300 \mathrm{kV}$. The free-standing graphene, which represents highly ordered graphene sheets, is imaged successfully on aberrationcorrected FEI Titan Cube TEM at $80 \mathrm{kV}$ (FEI Titan $3^{\mathrm{TM}}$ G2 60 300, 2009 [Internet]).

\section{RESULTS AND DISCUSSION}

Atomic resolution imaging of graphene layers is very difficult in any conventional microscopes because a higher $\mathrm{kV}$ beam damages the thinnest carbon layers and a lower $\mathrm{kV}$ operation deteriorates resolution and sensitivity for the light atoms. The aberration-corrected TEM operating at a low $\mathrm{kV}$,
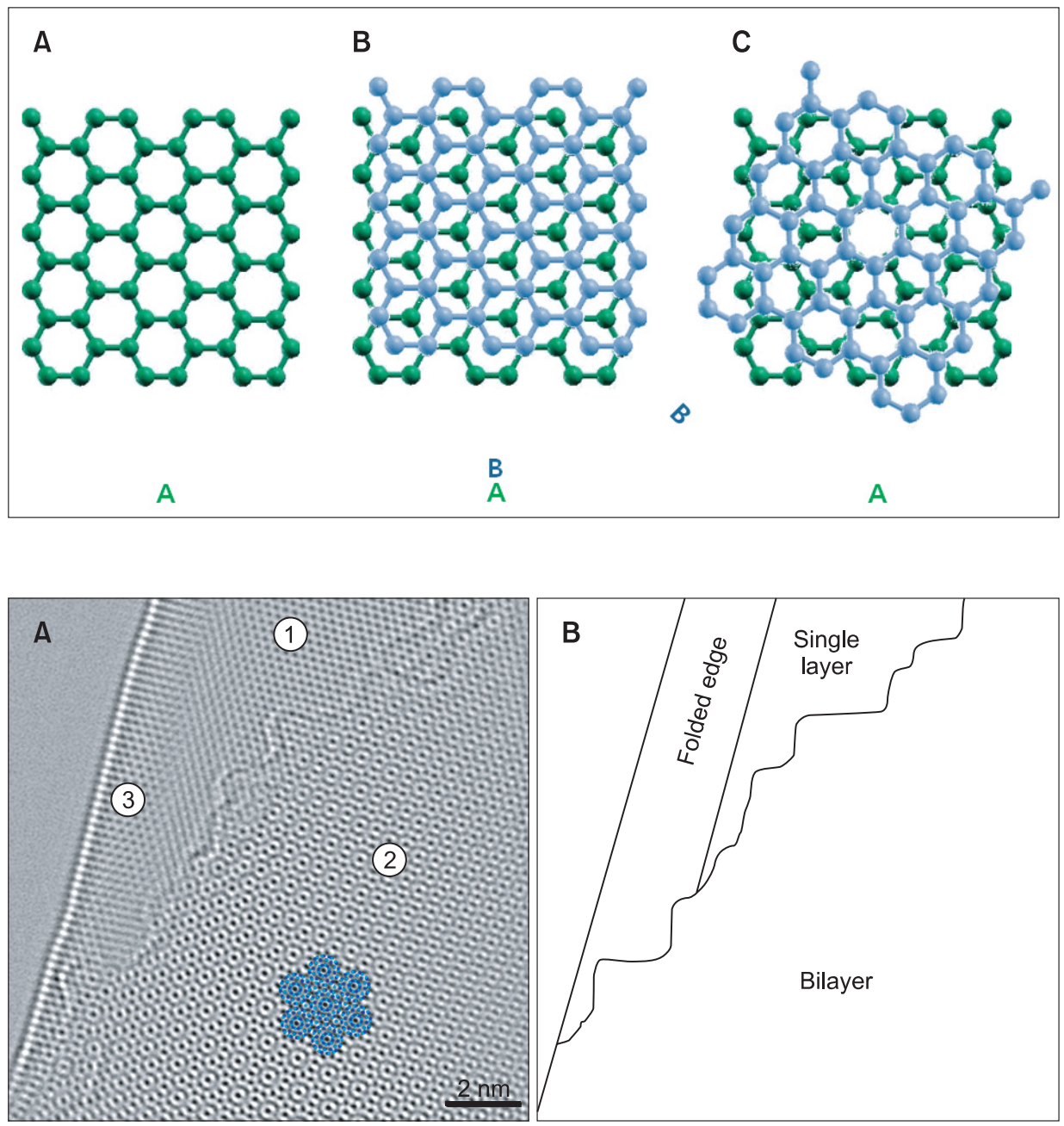

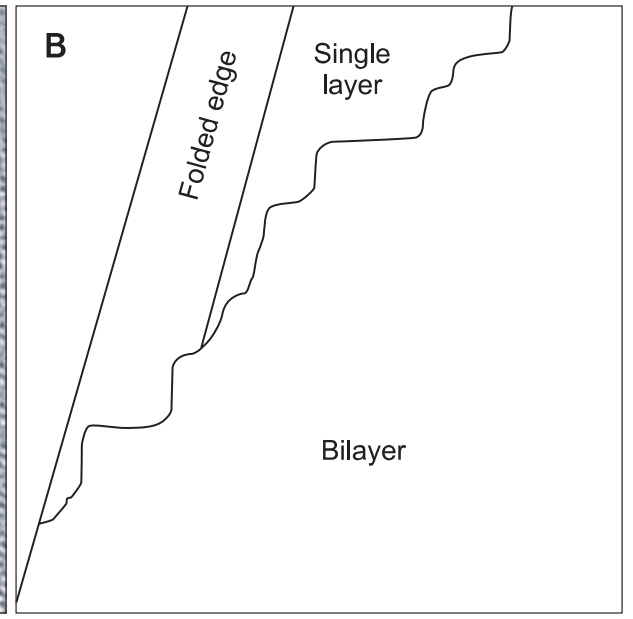

Fig. 1. The atomic models of single and bilayer graphene configuration. Green and light blue atoms indicate individual carbon atoms on top of each other, respectively. (A) Single layer graphene, (B) Graphitic A and B layers indicate Bernalstacked bilayer graphene, (C) Stacking structure of rotated bilayer graphene. The rotation angle 45 degree and is equivalent to 15 degree rotation.
Fig. 2. Exit wave reconstructed phase image (A) of overlapped free-standing graphene sheet and corresponding carbon atom positions overlaid. The bilayer region indicates a Moiré pattern. There are regions of folded edge, single layer and bilayer graphene sheet as indicated in the schematic (B). 
Single layer

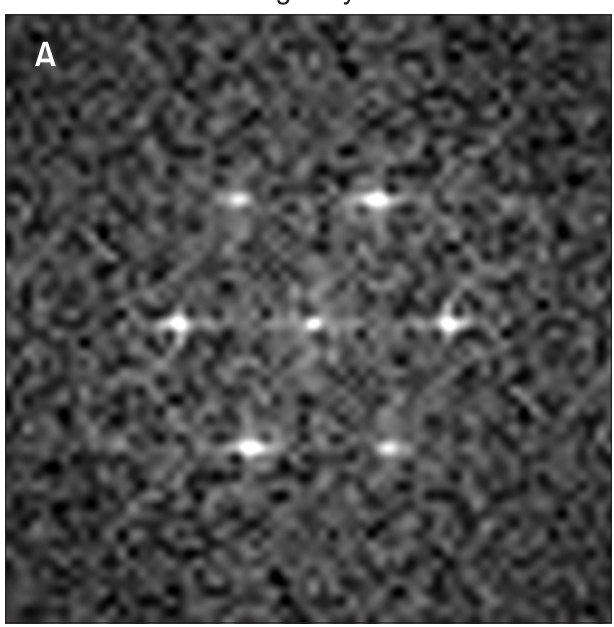

Bilayer

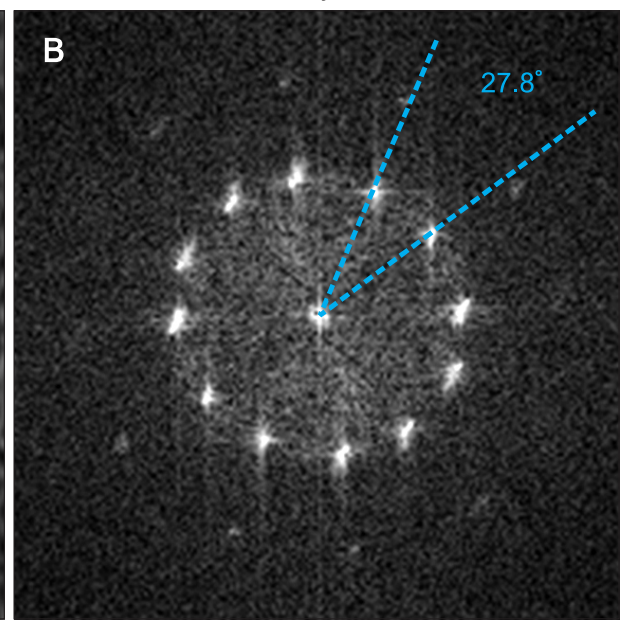

Fig. 3. (A) The digital diffractograms from single layer and bilayer graphene regions from the regions 1 and 2 as marked in the Fig. 2. (B) Indicates the rotation angle 27.8 degree. however, simultaneously brings us a high resolving power and sensitivity even at $80 \mathrm{kV}$ for imaging and spectroscopy of the preserved graphene layers. The acceleration voltage $80 \mathrm{kV}$ is optimized for graphene atomic imaging because the electron energy is below $86 \mathrm{keV}$, the threshold knock-on damage of free-standing graphene sheet (Zobelli et al., 2007).

When single and bilayer graphene layers are considered, the atomic models with perfect single layer, graphitic Bernalstacked bilayer and rotated graphene bilayer are built as shown in Fig. 1. The green and light blue dots indicate individual carbon atoms on top of each other. While Fig. 1A indicates the perfect single layer graphene, Fig. $1 \mathrm{~B}$ represents graphitic stacked layers of A and B, so-called Bernal-stacked bilayer graphene and Fig. 1C is stacking structure of twisted bilayer graphene. As an example, the rotation angle 45 degree is illustrated and this angle is equivalent to 15 degree rotation in the other way around as well. This rotated graphene atomic model displays quite weird pattern even in short range due to the overlapping layers. This pattern is a Moiré pattern directly obtained from two identical hexagonal lattices, which represents two individual $\mathrm{sp}^{2}$ carbon layers.

A TEM image from a typical region of the synthesized free-standing graphene layer is shown in Fig. 2A. The exit wave reconstructed image of overlapped free-standing graphene sheets from focal series is overlaid with atomic model corresponding carbon atom positions. Exit wave reconstructions are performed from a through-focus series with fifteen images taken by increments of $2 \mathrm{~nm}$ defocus using MacTempasX software. This phase image shows the exact atom position of carbon. In this figure, it is evident that regions of single layer (1), bilayer graphene (2) and folded edge (3) as indicated in the schematic Fig. 2B. The Fig. 2 displays densely packed hexagonal single-layer of carbon and unambiguous Moiré patterns formed from superimposed two individual graphene layers, which may represent a

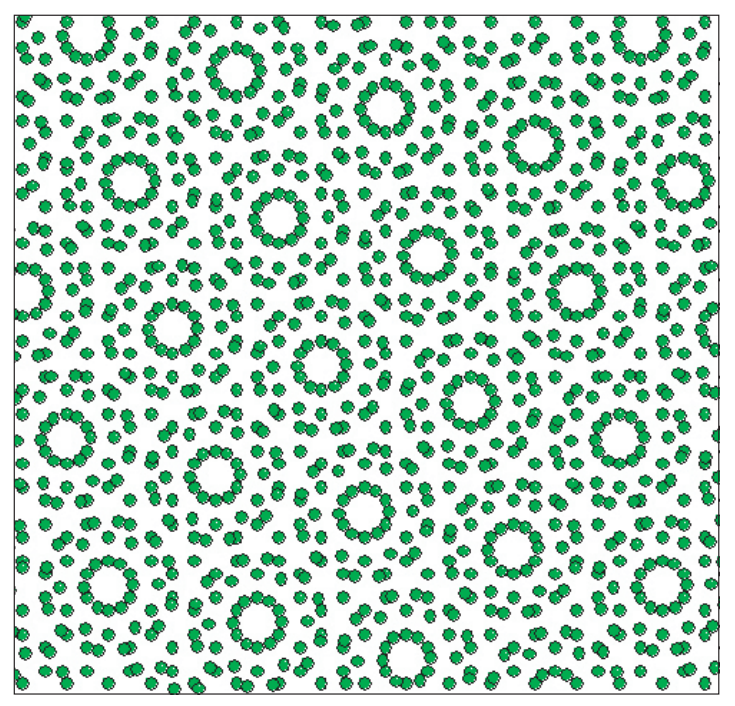

Fig. 4. An atomic model of overlapped graphene sheets with 27.8 degree rotation angle. This bilayer graphene model represents the region 2 in the Fig. 2.

coincidence site. Fig. 3A is Fourier transformed digital diffractogram from single layer graphene of region 1 in Fig. 2. The spots indicate just one set of hexagonal reflections out of single layer graphene. In contrast, Fig. 3B represents two sets of hexagonal reflections from two overlapping layers of graphene sheets. Especially this case is identified as a bilayer with a rotation angle, 27.8 degree. In order to confirm this stacking configuration, the atomic model of overlapped graphene sheets with 27.8 degree rotation angle is built as in Fig. 4. This bilayer graphene model corresponds to the region 2 in the Fig. 2. Using this model, the image simulation is performed with the condition of positive and negative defocus conditions as shown in Fig. 5B and D, respectively. The one shows bright atom contrast with a positive defocus 


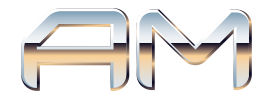

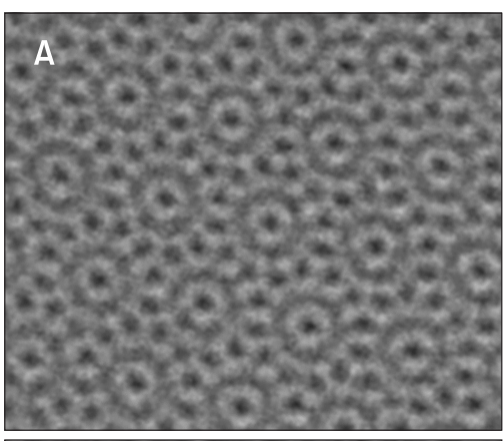
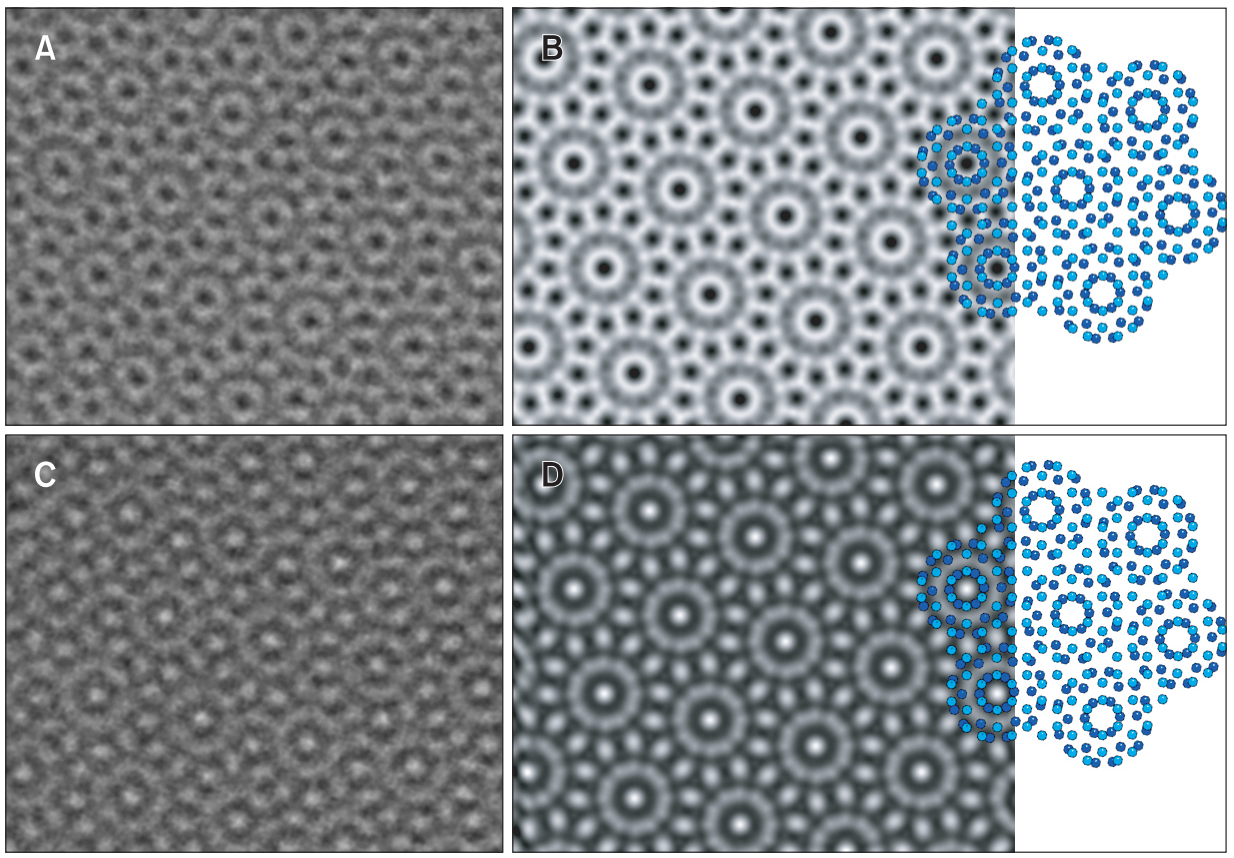

Fig. 5. The bilayer images with 27.8 degree rotation angle. Comparing real images taken and simulated images from the atomic model. (A) indicates the real image at $59 \mathrm{~nm}$ defocus and the corresponding simulated image of (B). (C) Indicates the real image at $-11 \mathrm{~nm}$ defocus and the corresponding simulated image of (D).

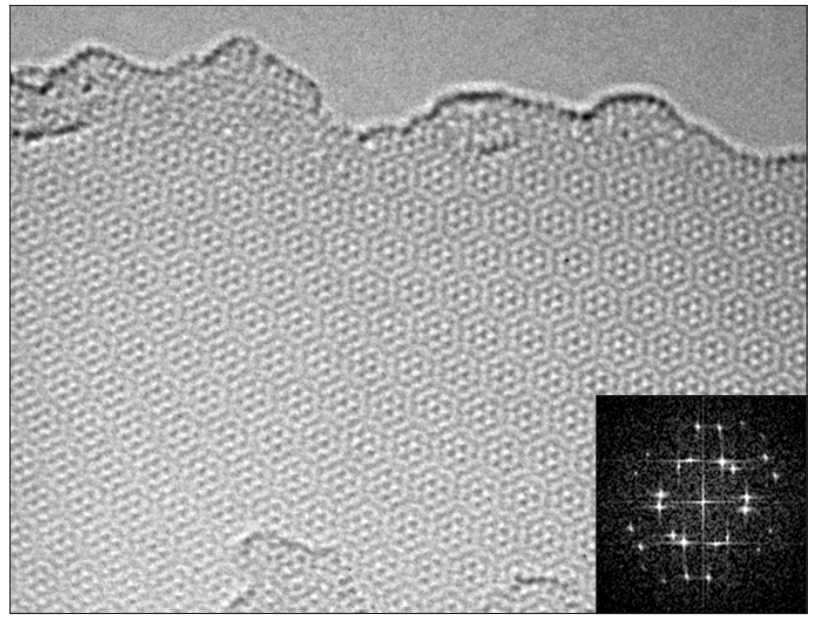

Fig. 6. The real aberration-corrected image displayes a bilayer graphene configuration with 17.9 degree rotation angle. The inset of digital diffractogram represents the bilayer with the rotation angle.

in a negative $\mathrm{C}_{\mathrm{s}}$ correction condition of about $-0.015 \mu \mathrm{m}$ and $\mathrm{C}_{5}$ of $5 \mathrm{~mm}$, while the other represents dark atom contrast in a negative defocus condition. The simulation results are correlated to the overplayed atom models as shown in Fig. 5B and $\mathrm{D}$ and confirmed with the corresponding real images Fig. $5 \mathrm{~A}$ and $\mathrm{C}$ taken at $59 \mathrm{~nm}$ and $-11 \mathrm{~nm}$ defocus, respectively, which shows bright and dark contrast Moiré patterns. The single raw images taken at proper defocus shows minimized delocalization because of minimized spherical aberration and are able to be correlated directly to the phase image in Fig. 2, which has coincident contrast at the equivalent atom positions. The results of comparison prove unambiguous Moiré pattern formation and contrast from the 27.8 degree rotated bilayer with atomic resolution imaging.

Monochromated electron energy loss spectra (EELS) of freestanding graphene sheets are also confirmed. The carbon $\mathrm{K}$-edge in EELS is used to identify the structure of graphene sheets and the EELS of single layer sheet exhibits unique graphitic characteristics, which has the $1 s-\pi^{*}$ and $1 s-\sigma^{*}$ transition at $285 \mathrm{eV}$ and $291 \mathrm{eV}$.

\section{CONCLUSIONS}

Aberration-corrected imaging with a proper acceleration voltage achieves direct atomic resolution study of single and bilayer graphene sheets. Bilayer of graphene is found in synthesized graphene sheets with various synthetic routes. The rotation angle in the twisted graphene bilayer can be identified by measuring the rotation angle from Fourier transformed digital diffractogram and directly from the electron diffraction with parallel beam. The comparison between the real image at a proper defocus with a negative $\mathrm{C}_{\mathrm{s}}$ correction value and simulation result should be required to confirm the atomic imaging and the Moiré patterns. The bright and dark atom contrast condition can be figured out with the aberration corrector optimization. Once the imaging condition is tuned, another Moiré patterns with different rotation angles is possible to be analyzed directly from single atomic resolution imaging. As additional case in Fig. 6, the real image indicates a bilayer graphene with a Moiré pattern formation with a rotation angle 17.9 degree, which is directly 
measured from the inset digital diffractogram. The stacking order of multilayer graphene, which is usually appeared in synthesized graphene, can be analyzed properly at atomic scale by using aberration-corrected electron microscopy at 80 $\mathrm{kV}$.

\section{ACKNOWLEDGMENTS}

I would like to recognize valuable discussion with Dato A, Radmilovic V, and Dahmen U.

\section{REFERENCES}

Abergel D S L and Fal'ko V I (2007) Optical and magneto-optical farinfrared properties of bilayer graphene. Physical Review B 75, 155430-155434.

Banhart F, Kotakoski J, and Krasheninnikov A V (2011) Structural defects in graphene. ACS Nano 5, 26-41.

Bao W, Jing L, Velasco Jr J, Lee Y, Liu G, Tran D, Standley B, Aykol M, Dronin S B, Smirnov D, Koshino M, McCann E, Bockrath M, and Lau C N (2011) Stacking-dependent band gap and quantum transport in trilayer graphene. Nature Physics 7, 948-952.

Castro Neto A H, Guinea F, Peres N M R, Novoselov K S, and Geim A K (2009) The electronic properties of graphene. Rev. Mod. Phys. 81, 109-162.

Dato A, Radmilovic V, Lee Z, Phillips J, and Frenklach M (2008) Substratefree gas-phase synthesis of graphene sheets. Nano Letters $\mathbf{8}, 2012-$ 2016.

FEl.com (2009) Titan3'TM G2 60-300 [Internet]. Available from: http:// www.fei.com/uploadedFiles/DocumentsPrivate/Content/titan_ cubed_g2_ds.pdf.

Kim K, Lee Z, Malone B D, Chan K T, Alemán B, Regan W, Gannett W, Crommie M F, Cohen M L, and Zettl A (2011a) Multiply folded graphene. Physical Review B 83, 245433-245440.

Kim K, Lee Z, Regan W, Kisielowski C, Crommie M F, and Zettl A (2011b) Grain boundary mapping in polycrystalline graphene. ACS Nano $\mathbf{5}$, 2142-2146.

Lee Z, Jeon K J, Dato A, Erni R, Richardson T J, Frenklach M, and Radmilovic V (2009a) Direct imaging of soft-hard interfaces enabled by graphene. Nano Letters $\mathbf{9}, 3365-3369$.

Lee Z, Dato A, Jeon K J, Erni R, Richardson T J, Frenklach M, and Radmilovic V (2009b) Atomic resolution imaging and spectroscopy of graphene using the TEAM 0.5. Microscopy \& Microanalysis 15, 124125.

Li X, Cai W, An J, Kim S, Nah J, Yang D, Piner R, Velamakanni A, Jung I, Tutuc E, Banerjee S K, Colombo L, and Ruoff R S (2009) Large-area synthesis of high-quality and uniform graphene films on copper foils. Science 324, 1312-1314.

Novoselov K S, Geim A K, Morzov S V, Jiang D, Zhang Y, Dubonos S V, Grigorieva I V, and Firsov A A (2004) Electric field effect in atomically thin carbon films. Science 306, 666-669.

Novoselov K S, Jiang D, Schedin F, Booth T J, Khotkevich V V, Morozov S $\mathrm{V}$, and Geim A K (2005) Two-dimensional atomic crystals. PNAS 102, 10451-1045.

Ohta T, Bostwick A, Seyller T, Horn K, and Rotenberg E (2006) Controlling the electronic structure of bilayer grapheme. Science 313, 951-954.

Park S and Ruoff R S (2009) Chemical methods for the production of graphenes. Nature Nanotechnology 4, 217-224.

Zhang Y, Tang T T, Girit C, Hao Z, Martin M C, Zettl A, Crommie M F, Shen $Y R$, and Wang F (2009) Direct observation of a widely tunable bandgap in bilayer graphene. Nature 459, 820-823.

Zobelli A, Gloter A, Ewels C, Seifert G, and Colliex C (2007) Electron knockon cross section of carbon and boron nitride nanotubes. Physical Review B 75, 245402 